\title{
PROGRAMA HORMONAL ASSOCIADO AO DESMAME TEMPORÁRIO, NA INDUÇÃO DE OVULAÇÃO EM VACAS DE CORTE DURANTE O PÓS-PARTO
}

\author{
HORMONAL PROGRAM ASSOCIATED TO TEMPORARY WEANING IN THE INDUCTION OF \\ OVULATION IN BEEF COWS DURING POST-PARTUM
}

\author{
Marlon Nadal Maciel ${ }^{1}$ Jairo Pereira Neves ${ }^{2}$ Paulo Bayard Dias Gonçalves ${ }^{3}$ João Francisco de Oliveira $^{4}$ \\ Ederson Bisognin Bortolotto ${ }^{5}$ Patrícia Stranieri $^{6}$
}

RESUMO

Este experimento foi desenvolvido com o objetivo de avaliar a eficiência de tratamentos hormonais, associados ao desmame temporário, na indução de ovulação após o parto, em fêmeas de corte criadas extensivamente. Foram utilizadas 143 vacas (Hereford e cruzas Hereford $x$ Nelore), pluríparas, entre 50 e 70 dias após o parto, com condição corporal (CC) 2 e 3 (1-5). O grupo SEMED (somatotropina, estradiol, medroxiprogesterona, gonadotrofina e desmame) foi constituído por 50 vacas, as quais receberam (dia 0) 500mg de somatotropina bovina recombinante (bST-r), $5 \mathrm{mg}$ de benzoato de estradiol e um pessário intravaginal contendo $250 \mathrm{mg}$ de acetato de medróxiprogesterona (MAP) e, seis dias após, (dia 6) 500UI de gonadotrofina coriônica eqüina (eCG). No momento da retirada dos pessários (dia 7), os terneiros foram separados totalmente das vacas por 96h. No grupo EMED (estradiol, medroxiprogesterona, gonadotrofina $e$ desmame), constituído de 48 vacas, adotou-se um tratamento semelhante ao do grupo anterior, diferindo apenas na não utilização da somatotrofina. No grupo CONTROLE, 43 vacas foram unicamente separadas dos seus filhos por 96h. Logo após a retirada dos pessários vaginais e de realizado o aparte dos terneiros, as vacas foram colocadas em um piquete com touros (1:10). Após 53 dias, foi realizado o diagnóstico de gestação por palpação retal e ultra-sonografia, para detectar as vacas que conceberam no estro subseqüente aos tratamentos. Obtiveram-se indices de prenhez de $11,1 \%, 38,0 \%$ e $56,2 \%$ respectivamente, para os grupos CONTROLE, SEMED e EMED, cujas diferenças foram significativas $(p<0,0001)$ entre os grupos. No grupo $S E M E D$, a CC das vacas interferiu no tratamento, pois foi obser- vada superioridade $(p<0,01)$ nos índices de prenhez de vacas com CC 3 quando comparadas com vacas em CC 2. Essa diferença não foi verificada nos demais grupos. Os resultados também demonstraram que os tratamentos agiram de maneira diferenciada em vacas com CC 2, com percentuais de prenhez de 12,5, 32,2 e $55,5 \%$, respectivamente, para os grupos CONTROLE, SEMED e EMED (SEMED vs CONTROLE = p <0,01; SEMED vs EMED $=p<0,01 ;$ CONTROLE vs EMED = p<0,0001). Os índices de prenhez das vacas com CC 3 foram 10,5, 50,0 e 55,0\%, respectivamente, para os grupos CONTROLE, SEMED e EMED e indicaram diferença $(p<0,0001)$ entre os dois grupos e o grupo controle. Os resultados evidenciaram que o tratamento sem a utilização da bST-r, dispensado ao grupo EMED, pode ser adotado com sucesso tanto em vacas em CC 2 como em vacas em CC 3 , diferindo do tratamento adotado no grupo SEMED, o qual mostrou-se eficiente somente em vacas com CC 3 .

Palavras-chave: anestro pós-parto, indução da ovulação, bovinos de corte.

\section{SUMMARY}

The purpose of this experiment was to assess the efficiency of hormonal treatments on the fertility of beef cows raised extensively on the west border region of Rio Grande do Sul, Brazil. A hundred and forty-three cows (Hereford and Crossing breeds) which were between 50 and 70 days after delivery were used and, after being classified according to their body condition $(B C)$, varying from 1 to 5 , they were separated into three groups. The SEMED group had 50 cows, which

\footnotetext{
${ }^{1}$ Médico Veterinário, Doutorando do Programa de Pós-graduação em Medicina Veterinária, Universidade Federal de Santa Maria (UFSM), Bolsista CNPq.

${ }^{2}$ Médico Veterinário, Doutor, Professor Titular, Departamento de Clínica de Grandes Animais, BioRep, UFSM, $97105-900$ Santa Maria, RS. E-mail: jpneves@lince.hcv.ufsm.br. Autor para correspondência.

${ }^{3}$ Médico Veterinário, PhD, Professor Titular, Departamento de Clínicas de Grandes Animais, UFSM.

${ }^{4}$ Médico Veterinário, Doutor, Professor Adjunto, Departamento de Clínicas de Grandes Animais, UFSM.

${ }^{5}$ Médico Veterinário, Mestre.

${ }^{6}$ Médico Veterinário, Ex-bolsista CNPq/PIBIC, UFSM.
} 
received (day 0) 500mg of bovine somatotropin (bST), 5mg of benzoate of estradiol and an intravaginal pessary containing $250 \mathrm{mg}$ of medroxiprogesteron acetate (MAP) and, six days later, (day 6), 500UI of equine chorionic gonadotropin (eCG). By the time the pessaries were withdrawn, the calves were totally separated from their mothers for 96 hours. In the EMED group, which had 48 cows, a treatment similar to the previous one was adopted, differing only in the non-use of somatotropin. In the CONTROL group, 43 cows were only separated from their calves for 96 hours. Right after the vaginal pessaries withdrawal and the separation from the calves, the cows were placed in an pasture with bulls (1:10). After 53 days, a pregnancy diagnosis via rectal palpation and ultrasonography was done to detect the cows that had conceived in the estrous subsequent to the treatment. Pregnancy rates of $11.1 \%, 38.0 \%$ and $56.2 \%$ were obtained for the groups CONTROL, SEMED e EMED respectively, whose differences were significant $(p<0.0001)$ among the groups. In the $S E M E D$ group the cows $B C$ interferred in the treatment, because a superiority in the pregnancy rates of cows with $B C 3$ was observed when compared to cows with $B C 2$. This difference was not verified in the other groups. The results also showed that the treatments had a different result for cows with $B C 2$, with pregnancy percentages of $12.5,32.2$ and 55.5 for the groups CONTROL, SEMED and EMED (SEMED vs CONTROL= $p<0.01 ;$ SEMED vs EMED = $p<0.01 ;$ CONTROL vs EMED = $p<0.0001)$. The pregnancy rates of cows with BC 3 were of 10.5 , 50 and 55\% respectively for the groups CONTROL, SEMED and EMED and they indicated a difference of $(p<0.0001)$ between both groups and the control group. The results evidenced that a treatment without the use of somatotropin, given to the EMED group, can be adopted successfully either in cows with BC 2 or in cows with $B C 3$, which is different from the treatment used in the SEMED group because it was efficient only with cows with BC 3 .

Key words: post-partum interval, estrous cycle induction, beef cows.

\section{INTRODUÇÃO}

Nos últimos anos, foram desenvolvidos vários trabalhos buscando alternativas para melhorar a eficiência reprodutiva dos rebanhos bovinos de corte, a partir da diminuição do intervalo entre partos, já que este se constitui em uma das principais causas da baixa produtividade dos sistemas de criação extensiva (KOCH, 1983). Para atingir o desejado intervalo entre partos de 365 dias, é necessário que as vacas concebam em no máximo 85 dias após o parto (STAGG et al., 1994). No Estado do Rio Grande do Sul, essa situação ocorre em apenas 20 a $25 \%$ das vacas pluríparas e em 6 a $15 \%$ das vacas primíparas. De acordo com as observações realizadas por diversos pesquisadores, a pecuária de corte gaúcha vem obtendo ao longo dos anos, baixos índices de produtividade em virtude das baixas taxas de natalidade.

Conforme SHORT (1990), o anestro pósparto, intervalo compreendido entre o parto e o primeiro estro subseqüente, tem o seu período de duração influenciado por vários fatores, com destacada importância para a lactação e para a condição nutri- cional do animal antes e após o parto, os quais estariam influenciando o controle endócrino do mesmo nesse período. Embora os mecanismos hormonais que atuam inibindo o reinício da atividade cíclica ovariana e retardando a recuperação da capacidade reprodutiva plena após o parto, ainda não sejam totalmente compreendidos na espécie bovina, algumas evidências indicam que a supressão da liberação de GnRH e a conseqüiente inibição dos pulsos de LH sejam os fatores endócrinos preponderantes na ocorrência de um período de anestro pós-parto prolongado (GREGG et al., 1986; WILLIAMS \& GRIFFITH, 1995). Estudos mais recentes têm também demonstrado que uma proteína derivada dos adipócitos, denominada leptina, além de ser um regulador do apetite, exerce importante papel na transmissão dos efeitos da condição metabólica do animal sobre sua fertilidade (FOSTER \& NAGATANI, 1999). Ainda, a dinâmica folicular, que na fêmea bovina obedece a um padrão de ondas durante o ciclo estral, denominadas ondas foliculares, as quais consistem em um processo seqüencial de recrutamento, seleção e dominância folicular, controlados pelo eixo hipotálamo-hipófise-ovariano, além de outros sistemas hormonais e fatores locais, produzidos no ovário (CAMPBELL et al., 1995), também exercem importante papel na retomada da capacidade reprodutiva após o parto. MURPHY et al. (1990) detectaram a presença do primeiro folículo dominante, em vacas com razoável condição corporal, em média 10,2 dias após o parto, porém a ovulação desse folículo ocorreu em somente $11,1 \%$ dos casos. Esse primeiro folículo dominante, detectado após o parto, possui receptores para LH e FSH nas células da granulosa, além de concentrações de progesterona, estradiol e testosterona no líquido folicular consideradas fisiológicas, o que sugere grande semelhança com folículos dominantes desenvolvidos durante a fase lútea do ciclo estral (BRADEN $\boldsymbol{e t} \boldsymbol{a l} ., 1986)$. Em vacas que não se encontram sob estresse nutricional, o longo período de anestro pós-parto se deve muito mais às falhas na ovulação dos primeiros folículos dominantes, causadas pela liberação insuficiente dos pulsos de LH, do que ao atraso ou ausência do seu desenvolvimento (MURPHY et al., 1990; ROCHE et at., 1992; STAGG et al., 1994). Apesar de consideráveis evidências indicando a existência de efeitos detrimentais da lactação sobre a retomada da capacidade reprodutiva, após o parto, na espécie bovina (CARRUTHERS \& HAFS, 1980; ODDE $\boldsymbol{e t}$ al., 1980; SPICER et al., 1986; PETERS \& LAMMING, 1990), os mecanismos envolvidos nessa regulação não são totalmente conhecidos. No entanto, a hipótese de que a sensibilidade do hipotálamo ao feed back 
negativo de estradiol seja potencializada pela ação dos opióides endógenos, estimulados pela amamentação, é bem aceita (SHORT et al., 1979; ACOSTA et al., 1983). Como conseqüência da liberação dos opióides, que atuam indiretamente sobre o hipotálamo, ocorre a diminuição da freqüência dos pulsos de GnRH e, conseqüentemente, de LH (ZALESKY et al., 1990).

Desde a década de sessenta, vários estudos têm demonstrado a eficiência do uso de progestágenos, associados ou não, a substâncias luteolíticas e gonadotróficas, na indução e sincronização do ciclo estral em rebanhos de corte. Com isso, o uso conjunto desses hormônios é realizado com o objetivo de antecipar ou recuperar a capacidade reprodutiva de fêmeas pré-púberes ou que estejam no período de anestro pós-parto. $\mathrm{O}$ tratamento de vacas de corte, durante o período pós-parto, com progestágeno, estrógeno ou ambos, parece ser eficiente na indução de estro, na formação do corpo lúteo e na ativação da ciclicidade ovariana (PRATT et al., 1982). O efeito resultante do uso de progestágeno, administrado por sete a nove dias, durante o anestro pósparto, traduz-se pelo aumento na concentração plasmática de progesterona anteriormente à primeira ovulação após o parto, a qual parece ser a responsável pela diminuição da ocorrência de ciclos estrais de curta duração, possibilitando ao corpo lúteo oriundo dessa primeira ovulação a plena atividade durante um período de tempo considerado fisiológico (COOPER $\boldsymbol{e t}$ al., 1991; RAMIREZ-GODINEZ $\boldsymbol{e t}$ al., 1982; DIMMICK et al., 1991; ROCHE et al. 1992; WERTH et al., 1996). Os percentuais de concepção no estro sincronizado, passaram de 27 para $56 \%$ quando as vacas receberam valerato de estradiol no momento da inserção do implante de progestágeno (PRATT et al., 1982). BO et al. (1996) relataram que o efeito supressor do estradiol exógeno sobre o crescimento do folículo dominante foi potencializado quando associado a progestágenos, provocando a atresia desse folículo e o surgimento de uma nova onda folicular em média 4,3 dias mais tarde. Afirmam ainda que os folículos originados dessa nova onda folicular podem responder a gonadotrofinas exógenas tão bem quanto os de uma onda folicular espontânea. ROCHE $\boldsymbol{e t}$ al. (1992) observaram comportamento estral em $85 \%$ das vacas tratadas com progestágeno por 7-12 dias e 400 a 800 UI de Gonadotrofina coriônica eqüina (eCG), sugerindo que esse tratamento possa ser adotado com sucesso em vacas no início do período pós-parto com baixa condição corporal. MULVEHILL \& SREENAN (1977), trabalhando com vacas com menos de 60 dias após o parto, relatam um aumento significativo nos índices de prenhez, quando do uso de eCG asso- ciado ao progestágeno, comparados com o uso exclusivo de progestágeno.

Esse trabalho buscou avaliar a eficiência de programas hormonais associados ao desmame temporário, na indução da ovulação em vacas de corte, criadas extensivamente, averiguando o incremento nos índices de concepção antes dos 80 dias após o parto, com o objetivo de oferecer subsídios para o manejo reprodutivo nesse tipo de animais.

\section{MATERIAL E MÉTODOS}

Este trabalho foi desenvolvido na Fazenda Santa Virgínia, localizada no município de Quaraí, Região da Fronteira-Oeste do Estado do Rio Grande do Sul, durante os meses de dezembro de 1998 e janeiro de 1999. Foram utilizadas 143 vacas pluríparas (Hereford e Hereford x Nelore), criadas em campo nativo que se encontravam entre 50 e 70 dias após o parto e em condição corporal (CC) 2 e 3 (15), sendo distribuídas em três grupos experimentais, de forma que cada grupo permanecesse com número semelhante de animais da mesma CC. Antes da formação dos grupos, todos os animais foram pesados e submetidos a exame ginecológico. O grupo SEMED foi constituído por 50 vacas, as quais receberam, no início do experimento (dia 0), o implante vaginal contendo $250 \mathrm{mg}$ de Acetato de medroxiprogesterona, 500mg de Somatotropina bovina recombinante (bST-r) e $5 \mathrm{mg}$ de Benzoato de estradiol. Seis dias após a realização desses procedimentos (dia 6), os animais receberam 500UI de eCG. No momento da retirada dos implantes vaginais (dia 7), as vacas foram separadas das crias, permanecendo assim por um período de $96 \mathrm{~h}$. No grupo EMED, formado por 48 vacas, foi instituído um programa semelhante ao adotado no grupo anterior, diferindo apenas pela não utilização da bST-r. No grupo CONTROLE, 43 vacas foram somente separadas dos seus filhos durante 96h. Logo após a retirada dos implantes vaginais e de realizado o desmame, as vacas foram colocadas em um piquete com touros (dia 7), na relação de 1:10, onde permaneceram por 70 dias. Passados 53 dias da introdução dos touros (dia 60), foi realizado o diagnóstico de gestação, pela palpação retal e ultra-sonografia. Ao final do experimento, juntamente com a retirada dos touros, as vacas foram submetidas a uma nova pesagem, para acompanhamento do peso das mesmas durante o experimento. Os custos com a administração dos medicamentos foram de aproximadamente U\$ 3.50 para o grupo EMED e U\$ 7.00 para o grupo SEMED (cálculos realizados em dólares considerando-se os valores de janeiro de 1999). A avaliação dos índices de resposta estral e de prenhez, nos diferentes tratamentos, foi 
realizada pelo teste de qui-quadrado $\left(\mathrm{X}^{2}\right)$, utilizandose o programa estatístico SAS (1988). A análise de contraste foi utilizada nas situações em que experimentos com mais de dois tratamentos apresentaram diferença estatística.

\section{RESULTADOS E DISCUSSÃO}

Os resultados obtidos mostraram índices de prenhez de $11,1 \%, 38,0 \%$ e $56,2 \%$ (Figura 1), respectivamente para os grupos CONTROLE, SEMED e EMED. Quando comparadas estatisticamente, foram detectadas diferenças entre as taxas de prenhez dos três grupos experimentais (EMED vs SEMED $\mathrm{p}<0,01$; EMED vs CONTROLE $\mathrm{p}<$ 0,0001; SEMED vs CONTROLE $p<0,0001)$. A idade gestacional, no momento da realização do diagnóstico de gestação, indicava que as vacas prenhes conceberam na primeira ovulação subseqüente aos tratamentos, sugerindo que os animais que não responderam aos tratamentos, possivelmente, tenham continuado em anestro durante o período de exposição aos touros. No grupo SEMED, foi verificado o efeito da CC sobre o tratamento (Figura 2), pois as vacas com $\mathrm{CC} 3$ obtiveram um índice de prenhez superior $(\mathrm{p}<0,01)$ ao das vacas com CC 2 . Essa diferença não foi verificada nos demais grupos (Figura 2). Os resultados também mostraram a influência dos tratamentos sobre as vacas em CC 2, com percentuais de prenhez de $12,5,32,2$ e $55,5 \%$, respectivamente, para os grupos CONTROLE, SEMED e EMED (EMED vs SEMED p < 0,01; SEMED vs CONTROLE $p<0,01$; EMED vs CONTROLE $p<$ 0,0001). Nesse caso, em comparação com o grupo

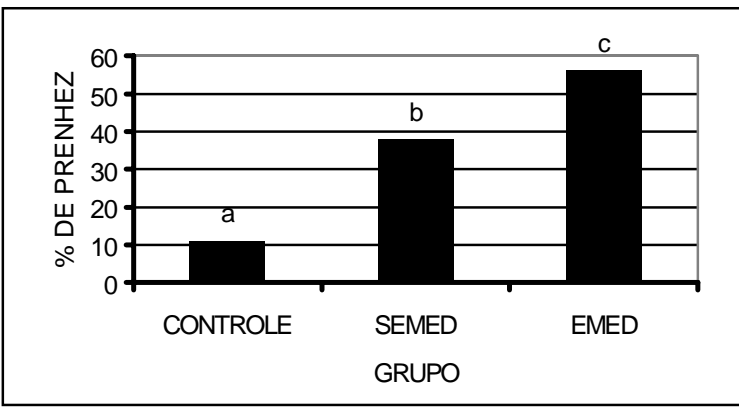

Figura 1 - Diferença entre os índices de prenhez obtidos em um total de 143 vacas Hereford e Hereford x Nelore, pluríparas, criadas em campo nativo, que se encontravam entre 50 e 70 dias após o parto com escore corporal 2 e 3 ( 1 = caquética e 5 = obesa), distribuídas nos seguintes grupos experimentais: CONTROLE (desmame), SEMED (somatotrofina, progestágeno, estradiol, gonadotrofina coriônica eqüina e desmame) e EMED (progestágeno, estradiol, gonadotrofina coriônica eqüina e desmame).

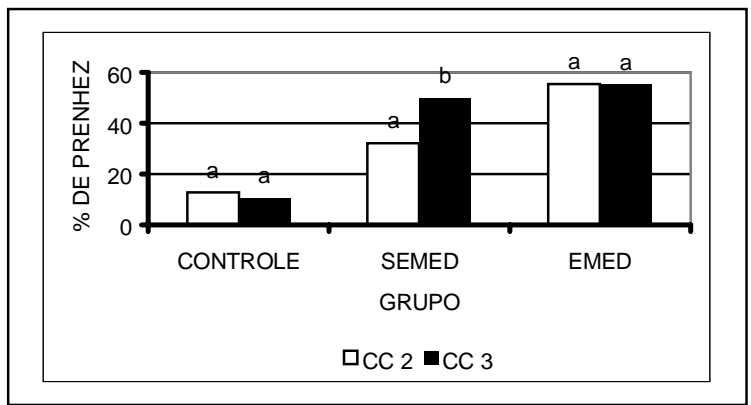

Figura 2 - Diferença entre os índices de prenhez obtidos nas vacas com condição corporal 2 e nas vacas com condição corporal 3 dentro de cada grupo experimental. Os grupos CONTROLE (desmame), SEMED (somatotrofina, progestágeno, estradiol, gonadotrofina coriônica eqüina e desmame) EMED (progestágeno, estradiol, gonadotrofina coriônica eqüina e desmame) foram constituídos por um total de 143 vacas Hereford e Hereford x Nelore, pluríparas, criadas em campo nativo, se que encontravam entre 50 e 70 dias após o parto.

EMED, os animais do grupo SEMED parecem ter tido o seu desempenho reprodutivo afetado, de forma direta ou indireta, pela administração da bST-r. Já em vacas com CC 3, os índices de prenhez de $50 \%$ do grupo SEMED, contra $55 \%$ do grupo EMED, não indicaram diferença $(p=0,47)$ entre os tratamentos. Entretanto, quando comparados com o grupo CONTROLE $(10,5 \%)$ foi observada diferença $(\mathrm{p}<0,0001)$.

Os baixos índices de prenhez, detectados no grupo CONTROLE, indicam que somente a realização do desmame durante $96 \mathrm{~h}$ não foi suficiente para induzir a ciclicidade estral e elevar os índices de prenhez a taxas satisfatórias, considerando-se os percentuais médios de repetição de cria (20 a 25\%) alcançados no Rio Grande do Sul, em propriedades onde não é adotado nenhum tipo de manejo visando ao incremento da eficiência reprodutiva. Esses dados sugerem que houve falta de equilíbrio nutricional necessário para que os animais respondessem aos efeitos causados pelo desmame, apesar de o ganho médio de peso das vacas durante o experimento ter sido de $300 \mathrm{~g} /$ dia. Em contrapartida, os percentuais de prenhez alcançados nos grupos SEMED e EMED indicam que a administração do progestágeno e do estradiol, ao mesmo tempo, parece ter sido eficiente em estimular a ciclicidade estral em um número considerável de animais, a exemplo do que foi descrito por ROCHE (1974) e BO et al. (1996). Esses dados indicam que vacas em anestro pós-parto, ao serem submetidas a tratamentos com associação hormonal anteriormente à primeira ovulação, desde que apresentem condições metabólicas compatíveis com a 
retomada da atividade reprodutiva, podem ter o intervalo de anestro pós-parto abreviado.

Da mesma forma, ainda baseando-se nos índices de prenhez do grupo EMED, o eCG parece ter sido efetivo na indução da ovulação, tanto em vacas com CC 2, teoricamente consideradas em condições metabólicas inferiores, como em vacas com CC 3, elevando o percentual de fêmeas que conceberam antes dos 80 dias após o parto para mais de 55\%. Assim, os achados assemelham-se aos de MULVEHILL \& SREENAN (1977), ROCHE $\boldsymbol{e}$ t al. (1992) e KASTELIC et al. (1997) que citam a boa eficiência na indução da ovulação, quando do uso de eCG após tratamento com progestágeno ou progesterona, em animais que se encontram em anestro pósparto. Dessa maneira, esse método pode constituir-se em um caminho alternativo para o incremento da eficiência reprodutiva e da produtividade em rebanhos bovinos de corte, considerando-se as taxas de prenhez e os custos do tratamento por animal.

\section{CONCLUSÃO}

A associação da bST-r aos programas de indução do ciclo estral após o parto, à base de progestágeno, estradiol, eCG e desmame, não é eficiente em incrementar a eficiência reprodutiva de fêmeas com condição corporal inferior a $3(1-5)$.

A utilização de eCG associada a progestágeno, estradiol e desmame pode ser eficiente em incrementar os índices de prenhez em vacas de corte, criadas em regime extensivo, antes dos 80 dias após o parto.

\section{REFERÊNCIAS BIBLIOGRÁFICAS}

ACOSTA, B., TARNAVSKY, T.E., PLATT, T.E., et al. Nursing enhances the negative effect of estrogen on $\mathrm{LH}$ release in the cow. Journal of Animal Science, v.57, p.1530-1536, 1983.

BO, G.A., ADAMS, G.P., PIERSON, R.A., et al. Effect of progestogen plus estradiol-17 $\beta$ treatment on superovulatory response in beef cattle. Theriogenology, v.45, p.897-910, 1996.

BRADEN, T.D., MANNS, J.G., CERMAK, D.L., et al. Follicular development following parturition and during the estrous cycle in beef cows. Theriogenology, v.25, p.833-843, 1986

CAMPBELL, B.K., SCARAMUZZI, R.J., WEBB, R. Control of antral follicle development and selection in sheep and cattle. Journal of Animal Science, v.49, p.335-350, 1995.

CARRUTHERS, T.D., HAFS, H.D. Suckling and four-times daily milking: influence on ovulation, estrus and serum luteinizing hormone, glucocorticoids and prolactin in postpartum Holsteins. Journal of Animal Science, v.50, p.919925,1980
COOPER, D.A., CARVER, C.A., VILlENEUVE, P., et al. Effects of progestogen treatment on concentrations of prostaglandin and oxytocin in plasma from the posterior vena cava of postpartum beef cows. Journal of Reproduction and Fertility, v.91, p.411-421, 1991.

DIMMICK, M.A., GIMENEZ, T., SPITZER, J.C. Ovarian endocrine activity and development of ovarian follicles during the postpartum interval in beef cows. Animal Reproduction Science, v.24, p.173-183, 1991.

FOSTER, D.L., NAGATANI, S. Physiological perspectives on leptin as a regulator of reproduction: Role in timing puberty. Biology of Reproduction, v.60, p.205-215, 1999.

GREGG, D.W., MOSS, G.E., HUDGENS, R.E., et al. Endogenous opioid modulation of luteinizing hormone and prolactin secretion in postpartum ewes and cows. Journal of Animal Science, v.63, p.838-847, 1986.

KASTELIC, J.P., OLSON, W.O., MARTINEZ, M. Sincronização do estro em bovinos Hereford-Angus com Crestar. Revista Brasileira de Reprodução Animal, v.21, p.101-103, 1997.

KOCH, R.M., ALGEO, J.W. The beef cattle industry: Changes and challenges. Journal of Animal Science, v.57, p.28-34, 1983.

MULVEHILL, P., SREENAN,J. Improvement of fertility in postpartum beef cows by treatment with PMSG and progestogen. Journal of Reproduction and Fertility, v.50, p.323-325, 1977.

MURPHY, M.G., BOLAND, M.P., ROCHE, J.F. Pattern of follicular growth and resumption of ovarian activity in postpartum beef suckler cows. Journal of Reproduction and Fertility, v.90, p.523-533, 1990.

ODDE, K.G., WARD, H.S., KIRACOFE, G.H., et al. Short estrous cycles and associated serum progesterone levels in beef cows. Theriogenology, v.14, p.102, 1980.

PETERS, A.R., LAMMING, G.E. Lactational anoestrus in farm animals. Oxford Reviwe Reproduction Biology, v.12, p.245-288, 1990.

PRATT, B.R., BERARDINELLI, J.G., STEVENS, L.P. Induced corpora lutea in the postpartum beef cow. I. Comparison of gonadotropin releasing hormone and human chorionic gonadotropin and effects of progesterone and estrogen. Journal of Animal Science, v.54, p.822-830, 1982.

RAMIREZ-GODINEZ, J.A., KIRACOFE, G.H., CARNAHAM, D.L., et al. Evidence for ovulation and fertilization in beef cows with short estrous cycle. Theriogenology, v.17, p.409413, 1982.

ROCHE, J.F. Effect of short-term progesterone treatment on estrous response and fertility in heifers. Journal of Reproduction and Fertility, v.40, p.433, 1974.

ROCHE, J.F., CROWE, M.A., BOLAND, M.P. Postpartum anoestrus in dairy and beef cows. Animal Reproduction Science, v.28, p.371-378, 1992.

SAS Statiscal Analysis Sistem. 3ed. Cary : SAS Institute, 1988. 1028 .

SHORT, R.E., RANDEL, R.D., STAIGMILLER, R.B., $\boldsymbol{e} \boldsymbol{t} \boldsymbol{a l}$. Factors affecting estradiol-induced LH release in the cow. Biology of Reproduction, v.21, p.683-684, 1979. 
SHORT, R.E. Physiological mechanisms controlling anestrous and infertility in postpartum beef cattle. Journal of Animal Science, v.68, p.799-816, 1990.

SPICER, L.J., LEUNG, K., CONVEY, E.M., et al. Anovulation in postpartum suckled beef cows. I. Associations among size and numbers of ovarian follicles, uterine involution, and hormones in serum and follicular fluid. Journal of Animal Science, v.62, p.734-741, 1986

STAGG, K., DISKIN, M.G., SREENAN, J. M., et al. Follicular development in long-term anoestrous suckled beef cows fed two levels of energy postpartum. Animal Reproduction Science, v.38, p.49-61, 1994.
WERTH, L.A., WHITTIER, J.C., AZZAM, S.M., $\boldsymbol{e}$ t al. Relationship between circulating progesterone and conception at the first postpartum estrous in young primiparous beef cows. Journal of Animal Science, v.74, p.616-619, 1996.

WILLIAMS, G.L., GRIFFITH, M.G. Sensory and behavioural control of gonadotrophin secretion during suckling-mediated anovulation in cows. Journal of Reproduction and Fertility, v.49, p.464-475, 1995.

ZALESKY, D.D, FORREST, D.W., McARTHUR, N.H., $\boldsymbol{e}$ t al. Suckling inhibits release of luteinizing hormone-releasing hormone from the bovine median eminence following ovariectomy. Journal of Animal Science, v.68, p.444-448, 1990

Ciência Rural, v. 31, n. 3, 2001. 